\title{
基于机器学习的电子束选区熔化成形件密度预测
}

\author{
元欣波 ${ }^{1}$ 李长鹏 ${ }^{2}$ 李 阳 ${ }^{3}$ 林 峰 ${ }^{3}$ 李 勇 ${ }^{1}$ 程 宣 $^{2}$ 陈国锋 $^{2}$ \\ (1. 清华大学摩擦学国家重点实验室 北京 100084; \\ 2. 西门子中国研究院 北京 100102 ;
}

3. 清华大学生物制造与快速成形技术北京市重点实验室 北京 100084)

\begin{abstract}
摘要: 电子束选区熔化技术, 以其快速加工复杂几何形状、真空熔炼、成形件残余应力小、粉末可回收等优点, 是近年来快 速发展的一种增材制造技术。但该技术加工参数众多, 目前很难建立起直接且准确的加工参数与成形件性能的关系。通过调 节电子束选区熔化技术的扫描速度、束流、基板温度、粉层厚度等四种参数, 制备了一系列 Inconel718 立方体样品, 并测 试获得其密度性能。利用线性回归、支持矢量回归和神经网络等三种机器学习算法建立了四种加工参数与密度的关系。算法 结果表明: 线性回归因其模型容量小, 预测精度最差; 神经网络模型容量大, 但易出现过拟合, 预测精度较好; 支持矢量回 归的模型容量适当, 且解释性良好, 预测精度最高。
\end{abstract}

关键词: 机器学习; 电子束选区熔化; 增材制造; 密度; 预测

中图分类号: TP181

\section{Machine Learning Algorithms on Density Prediction of Electron Beam Selective Melted Parts}

\author{
QI Xinbo $^{1} \quad$ LI Changpeng $^{2} \quad$ LI Yang $^{3} \quad$ LIN Feng $^{3} \quad$ LI Yong $^{1}$ \\ CHENG Xuan ${ }^{2}$ CHEN Guofeng ${ }^{2}$ \\ (1. State Key Laboratory of Tribology, Tsinghua University, Beijing 100084; \\ 2. Corporate Technology, Siemens Ltd., Beijing 100102; \\ 3. Bio-manufacturing and Rapid Forming Technology Key Laboratory of Beijing, \\ Tsinghua University, Beijing 100084)
}

\begin{abstract}
Electron Beam Selective Melting (EBSM) is a novel additive manufacturing technology, which is developing very fast nowadays. It has many advantages: building up parts with complex morphology; processing under vacuum to get rid of the impurity; its manufactured part with small residual stress and powder recycling. However, it is difficult to establish the relationship between EBSM's processing parameters and parts' properties. Here a series of Inconel 718 cubic specimens are manufactured through adjusting EBSM's scanning speed, beam current, plate temperature and layer thickness. Then the densities of these specimens are measured. Three kinds of machine learning algorithms, including linear regression, support vector regression and neural network, have been utilized to build the relationship between these four processing parameters and density. The results show that: Linear regression has the worst prediction skill as a result of its small model capacity; neural network has a better prediction accuracy, but it is easily overfitting; support vector regression has appropriate model capacity and good physical interpretation, and it behaves best in the density prediction.
\end{abstract}

Key words: machine learning; electron beam selective melting; additive manufacturing; density; prediction

\section{0 前言}

电子束选区熔化技术是近年来快速发展的一种
增材制造技术，其通过高速电子轰击金属粉末，产 生的动能转化成热能来熔化金属粉末，从而逐层打 印出所需形状的构件 ${ }^{[1-2]}$ 。电子束选区熔化技术有如 下主要优点：(1) 相对于传统制造工艺，如铸造、锻 造等，其可直接加工复杂几何形状，因此非常适用 于小批量复杂零件的直接量产 ${ }^{[3]}$; (2) 粉末在真空中 
熔融并成形, 故可避免在空气中熔融所带来的氧化 缺陷等质量事故 ${ }^{[4]}$; (3) 原材料利用率高, 零件可近 净成形, 后续加工量小。粉末利用率高, 打印后未 被熔化的粉末可以回收再利用。但该技术也有很多 局限性需要突破, 如吹粉和元素蒸发、表面质量不 高、成形尺寸较小、需要真空环境等 ${ }^{[5]}$ 。

电子束选区熔化过程的工艺参数复杂, 如电子 束电流、加速电压、扫描速度、基板温度、作用时 间、粉层厚度、扫描方式等因素都对成形件的性能 影响巨大 ${ }^{[6-7]}$ 。建立工艺参数组合与材料性能之间的 对映关系, 对于加工制造高质量的成形件具有决定 性作用。然而, 电子束选区熔化过程涉及多尺度(微 观尺度的电子束与粉末作用、介观尺度的固液相变 及宏观尺度的变形)和多物理场(温度场、流场及应 力场等)相互作用, 使用传统的解析计算和数值模拟 方法较难准确且高效地再现这一过程。近年来, 机 器学习算法作为一种数据驱动的建模方法, 已经开 始应用于增材制造工艺规划中。SHEN 等 ${ }^{[8]}$ 使用基 于反向传播的神经网络算法研究了选区激光烧结技 术的激光功率、扫描速度、扫描间距和粉层厚度这 四个工艺参数对烧结密度的影响, 对比结果表明神 经网络算法与试验结果吻合地很好; ZHANG 等 ${ }^{[9]}$ 同样基于反向传播神经网络算法研究了电子束选区 熔化技术的铺粉器平移速度和旋转速度对单位宽度 单位时间内粉末平铺体积和表面粗糙度的影响, 且 该算法与传统的离散元模拟相结合, 解决了神经网 络学习数据来源的问题。蔡从中等 ${ }^{[10]}$ 使用支持矢量 回归算法建立了选区激光烧结的工艺参数(层厚、扫 描间距、激光功率、扫描速度、加工环境温度、层 间加工时间间隔和扫描方式)与成形件密度间的预 测模型, 证明了支持矢量回归对于解决该类问题的 有效性。

密度是材料致密程度的重要参数表征, 其大小 能够显著影响制件的强度、塑性及疲劳裂纹扩展等 力学行为。研究表明 ${ }^{[1-12]}$, 密度越大, 即材料越致 密, 使得内部孔洞越少, 此时能够有效地减小由于 微孔造成的应力集中和裂纹萌生, 从而提高制件的 机械性能。因此, 本文选择密度值作为研究目标, 探究电子束选区熔化技术的工艺参数与密度参数 之间的对应关系。具体地, 首先控制电子束选区熔 化成形技术的四种工艺参数: 扫描速度、束流强 度、基板温度和粉层厚度, 制备了一系列 Inconel718 样品, 并测试获得相应密度, 然后分别 基于线性回归、支持矢量回归和神经网络三种算法 建立了工艺参数与密度之间的预测模型, 并进行了 详细的性能对比。

\section{1 试验制备}

试验采用天津清研智束科技有限公司生产的 QbeamLab 设备, 该设备主要由操作平台(触摸屏等 计算机外设)、电气控制柜(工业控制计算机、振粉 驱动器、可编程序控制器等)和真空控制柜(真空室、 伺服电机等)三部分组成。

试验研究了扫描速度(范围 $600 \sim 3000 \mathrm{~mm} / \mathrm{s}$ )、 束流(范围 6 26 mA)、基板温度(范围 1 026.5 $1128.5 \mathrm{~K}$ )、粉层厚度(范围 $50 \sim 75 \mu \mathrm{m}$ ) 这四个工艺 参数, 粉末采用 Additive Metal Alloys Limited 公司生 产的 Inconel718 合金粉末，粉末尺寸为 $45 \sim 105 \mu \mathrm{m}$ 之间。如上工艺参数的选择范围是经过前期试验 探索后得到的能够使得金属制件稳定成形的加工 窗口。其他的打印参数均固定不变, 具体数值见 表 1 。

表 1 QBeamLab 电子束选区熔化设备打印参数

\begin{tabular}{lc||cc}
\hline \multicolumn{1}{c|}{ 设备参量 } & 数值 & 设备参量 & 数值 \\
\hline 样品尺寸 $/ \mathrm{mm}^{3}$ & $15 \times 15 \times 10$ & 成形空间 $/ \mathrm{mm}^{2}$ & $100 \times 100$ \\
样品布局 & $4 \times 4$ & 样品间距 $/ \mathrm{mm}$ & 5 \\
焦点偏移量 & 0 & 扫描间距 $/ \mathrm{mm}$ & 0.15 \\
加速电压 $/ \mathrm{kV}$ & 60 & & \\
\hline
\end{tabular}

打印态样品如图 1 所示, 样品表面质量较好, 仅个别样品保留了较明显的熔池形貌。因为本试验 目的是建立工艺参数组合与密度的关系, 需要尽可 能大范围地探究极限工艺条件的影响, 同时又需保 证基本的成形能力, 因此, 目前的打印结果是可以 接受的。成形后的样品从基板上切下后，通过阿基 米德密度仪 ${ }^{[1]]}$ 测量其密度, 选用的溶液为酒精, 目 的是为了减小表面张力的影响。测量结果如附录的 表 3 所示, 以下将基于该表中的 72 组试验数据进行 分析, 探讨四种工艺参数组合对密度性能的影响, 并建立定量化的机器学习算法。

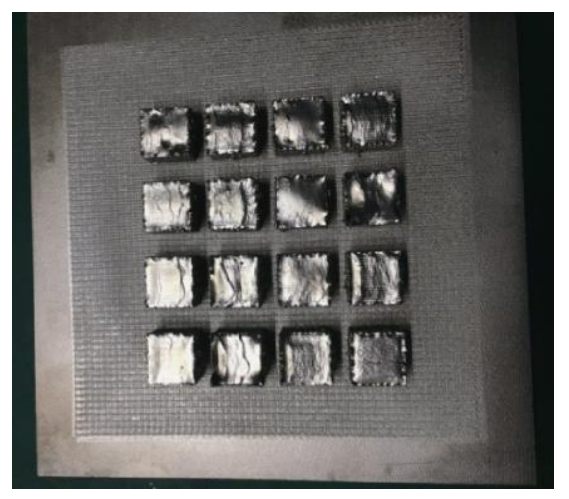

图 1 电子束选区熔化打印的样品形貌 


\section{2 机器学习算法}

\section{1 数据预处理}

机器学习算法是一种由数据驱动的数学分析 方法, 即算法质量强烈依赖于数据集的大小、分 布、特征等。图 2 描述了电子束选区熔化成形件 密度分布的统计结果。为了将数据处理成算法所需 要的形式, 需要对这里的原始数据进行一系列的预 处理操作。

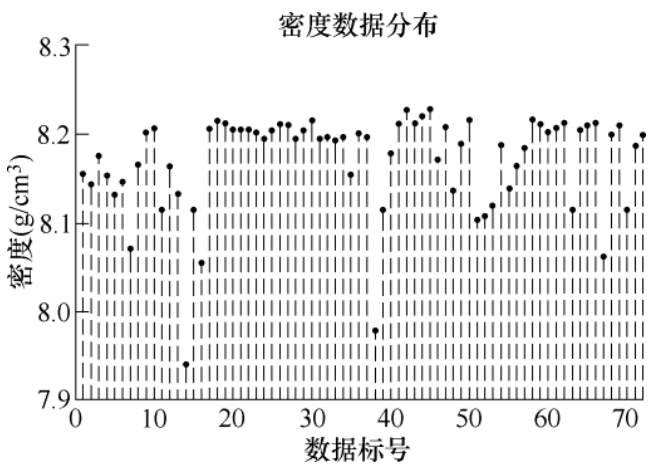

图 2 试验数据的统计分布

\subsection{1 非均衡数据集处理}

由图 2 可以看出, 大多数密度值浮动于 8.2 $\mathrm{g} / \mathrm{cm}^{3}$ 上下, 而密度为 $7.94 \mathrm{~g} / \mathrm{cm}^{3}$ 和 $7.97 \mathrm{~g} / \mathrm{cm}^{3}$ 的数 据量很小, 即该数据集对于密度值的大小是非均衡 的。如果不经过预处理, 增加低密度值占总数据量 的权重, 则机器学习算法通常会 “忽视” 这些数值, 导致对它们的预测精度降低。对于非均衡数据集, 已有很多种方法来处理, 比如采样法、代价敏感学 习和基于核函数的学习等 ${ }^{[12]}$ 。其中, 采样法是最简 单易行的一种方式, 其又分为上采样和下采样, 前 者是通过增加少数类样本的数量来实现样本均衡, 后者则通过减少多数类样本的数量来实现均衡。此 处运用上采样法, 直接复制少数类样本形成多条记 录, 从而增加此类样本在总样本中的权重, 间接提 高模型在此类样本上的损失代价。具体地, 这里将 密度为 $7.94 \mathrm{~g} / \mathrm{cm}^{3}$ 和 $7.97 \mathrm{~g} / \mathrm{cm}^{3}$ 的数据条目分别复 制两次(过采样法的复制次数是一个超参数, 需根据 经验提前设定：复制次数过多易造成过拟合，次数 过少则效果不明显), 即在原数据集基础上新增四条 “低密度值” 的数据, 因此, 以下机器学习算法所 作用的数据集的样本数为 76 组。

\subsection{2 划分数据集}

机器学习算法所需的数据集至少有两种: 训练 集和测试集, 前者用于训练模型, 后者用于评估模 型性能。如前所述, 总数据集一共有 76 组数据, 这
里先将其随机打乱, 然后抽取 10 组数据作为测试 集, 剩余的 66 组数据作为训练集。

特别地, 在训练模型过程中, 还可以再次将训 练集划分出一部分作为验证集, 用于实时地评估模 型准确率, 目的是找到最优的超参数组合。这里, 我们采用 $K$ 折交叉验证方法 ${ }^{[13]}$, 其具体过程是： 将训练集平均划分为 $K$ 份子集，其中 $K-1$ 份子集作 为训练集训练模型，剩余的 1 份子集作为验证集用 于模型评估。该过程重复 $K$ 次，从而使得每一份子 集都有机会用作验证集。一般地，增大 $K$ 值能够提 供更好的训练结果，但同时会加大计算开销。为了 兼顾精度和效率，此处设定 $K$ 等于 5 。

\subsection{3 数据归一化}

如附录中的数据表 3 所示，不同的特征量有不 同的数值范围: 扫描速度和基板温度在 $10^{3}$ 量级, 束流强度在 $10^{1}$ 量级，而粉层厚度在 $10^{2}$ 量级。如 果按照原有量级进行计算，则扫描速度和基板温度 这两个特征的方差就会在计算误差时起决定性作 用, 从而在学习算法中占据主导位置, 导致学习器 并不能像期望的那样从其他特征中学习，使得模型 精度下降。因此, 这里采用 “数据归一化”, 使得不 同量级之间的特征处于同一数量级，在数值上形成 一定比较性, 可以大大提高算法的准确性。另一方 面, 数据归一化后, 机器学习模型使用梯度下降法 求最优解时, 最优解的寻优过程明显会变得平缓, 更容易正确的收敛到最优解。

这里采用的归一化方法为 “最小最大化法” [14]

$$
x_{\text {norm }}=\frac{x-x_{\min }}{x_{\max }-x_{\min }}(\max -\min )+\min
$$

式中, $x_{\text {norm }}$ 是归一化后的某数据点, $x$ 是对应的原 始数据点, $x_{\text {max }}$ 为样本数据的最大值, $x_{\text {min }}$ 为样本 数据的最小值, $\max$ 为归一化后的区间范围的最大 值， $\min$ 为归一化后的区间范围的最小值。这里没 有选择传统的 $[0 ; 1]$ 归一化区间，而是设置区间范围 为 $[0: 1 ; 0: 9]$, 目的是为了避免有些学习算法在零点 处无导数的问题。另外一点需要注意的是，用于缩 放的 “比例尺” 仅在训练集上计算获得, 然后再统 一将其用于训练集和测试集，这么做的原因是保证 事先完全不知道测试集的规模和大小，降低其对算 法的影响。

\section{2 算法描述}

本文试图解决的问题归结为构建四个工艺参数 与密度值之间的数学关系式, 用数学语言表示为: 自变量是四个工艺参数(扫描速度、束流、基板温度、 粉层厚度), 记为 $\vec{x}=\left\{x_{1}, x_{2}, x_{3}, x_{4}\right\}$, 因变量是密度值, 
记为 $\vec{y}$ 。本部分将详细介绍所使用的机器学习算法。

\section{2 .1 线性回归算法}

线性回归算法是最简单的一种回归算法, 其假 设自变量和因变量之间是线性相关关系, 表达式为

$$
\begin{gathered}
f(x)=w_{1} x_{1}+w_{2} x_{2}+w_{3} x_{3}+w_{4} x_{4}+b= \\
\boldsymbol{w}^{\mathrm{T}} \boldsymbol{x}+b
\end{gathered}
$$

式中, $\boldsymbol{w}=\left\{w_{1}, w_{2}, w_{3}, w_{4}\right\}$ 是各特征的权重系数, $b$ 是偏置或截距。算法的目的是求得合适的系数和偏 置, 使得实际观测数据和预测数据之间的残差平方 和最小

$$
\min \sum_{i=1}^{4}(f(x)-y)^{2}
$$

\subsection{2 支持矢量回归算法}

支持矢量回归算法, 是用于分类问题的支持矢 量机算法的扩展, 其同样是希望学得一个形如式 2 的回归模型, 使得 $f(x)$ 与 $y$ 尽可能接近。但支持矢 量回归算法的优化目标有所不同

$$
\begin{gathered}
\min \frac{1}{2}\|\boldsymbol{w}\|^{2}+C \sum_{i=1}^{4}\left(\eta_{i}+\eta_{i}\right) \\
\text { s.t. } \boldsymbol{w} \phi(x)+b-y_{i} \leqslant \varepsilon+\eta_{i} \\
y_{i}-(\boldsymbol{w} \phi(x)+b) \leqslant \varepsilon+\eta_{i} \\
\eta_{i} \geqslant 0 \quad \eta_{i} \geqslant 0 \quad i=1,2,3,4
\end{gathered}
$$

式中, $\vec{w} \phi(x)+b$ 是回归超平面, $\vec{w}$ 和 $b$ 分别是该超 平面的权重系数和截距, $\phi(x)$ 是将样本从原始空间 到一个更高维的特征空间的映射函数, 它使得样本 在这个特征空间内可以做线性回归。实际运算时会 通过内积操作 $\kappa\left(x_{i}, x_{j}\right)=\left\langle\phi\left(x_{i}\right), \phi\left(x_{j}\right)\right\rangle$ 将 $\phi(x)$ 转化 为核函数 $\kappa(\cdot, \cdot)$, 该函数的形式决定了将样本映射 到何种特征空间中，因此对回归结果影响巨大。 $\varepsilon$ 表示预测值和实际值之间可容忍的偏差, 即仅当两 者之间的差别绝对值大于 $\varepsilon$ 时才计算损失。 $\eta_{i}$ 和 $\eta_{i}$ 为第 $i$ 个样本的松弛常量, 用于表征该样本不满足 约束 $y_{i}-(\vec{w} \phi(x)+b)$ 的程度。 $C$ 为正则化常数, 也 称为惩罚系数, 代表对离群点带来的损失的重视程 度(离群点即 $\eta_{i}$ 和 $\eta_{i}$ 大于 0 的点), $C$ 越大, 代表对 损失的重视越大, $C$ 无穷大时, 表示迫使所有的样 本均满足上述约束。

因此，支持矢量回归是一种有坚实理论基础的 机器学习方法, 其不仅有利于研究人员 “有的放矢” 地调节参数以达到模型最优效果, 同时保证了模型 的可解释性, 有效地避免了 “黑盒效应”。

\subsection{3 神经网络算法}

神经网络算法有多种, 如多层感知机、卷积神
经网络、递归神经网络等, 后面两种网络算法的结 构复杂，多用于图像数据和时间序列数据的分析预 测, 因此此处所说的神经网络算法特指多层感知机。

具体地, 算法由三类网络层构成: (1) 输入层: 接收输入特征量; (2) 隐藏层: 对上一层(可以是输 入层或上一层隐藏层)的信号进行函数处理; (3) 输 出层: 对最后一层隐藏层的信号进行函数处理, 并 输出结果。其中, 隐藏层和输出层的功能神经元都 由两部分构成: 线性加权和非线性激活, 整体表达 式形如

$$
f(\vec{x})=g\left(\vec{w}^{T} \vec{x}+b\right)
$$

式中, $\vec{w}^{T} \vec{x}+b$ 是线性加权公式, 同时是全连接形式, 表明前一层的每个节点与该层的每个节点都一一连 接, 即把前一层的输出特征都综合起来。函数 $g(\cdot, \cdot)$ 是非线性激活函数, 可以有多种形式, 常用的有

$$
\begin{gathered}
\operatorname{Sigmoid}(k)=\frac{1}{1+\exp (-k)} \\
\operatorname{Tanh}(k)=\frac{2}{1+\exp (-2 k)}-1 \\
\operatorname{ReLU}(k)=\max (0, k)
\end{gathered}
$$

神经网络算法对于回归问题的优化目标也 有多种形式, 这里选择最小化均方根误差 $(n$ 为样本 个数, $y_{i}$ 为模型在第 $i$ 个样本上的预测值, $y_{i}$ 为第 $i$ 个样本的试验值

$$
\min \left(\sqrt{\frac{1}{n} \sum_{i=1}^{n}\left|y_{i}-y_{i}\right|}\right)
$$

\section{3 机器学习预测}

本文应用了两个基于 Python 语言的机器学习 框架编写和运行算法: scikit-learn ${ }^{[15]}$ 和 PyTorch ${ }^{[16]}$ 。 前者包含了众多传统机器学习算法, 且提供了大量 简单易用的工具用于数据挖掘和分析; 后者侧重于 神经网络算法的开发和应用，使得用户方便地设置 神经网络结构、超参数和优化算法等。因此, 这里 调用 scikit-learn 进行线性回归和支持矢量回归预测, 使用 PyTorch 进行神经网络预测。该部分的第 3.1、 3.2 和 3.3 节分别对三种算法训练得到的具体模型参 数和训练结果进行概述, 第 3.4 节则详细定量对比了 这三种算法的性能, 第 3.5 节则对如何应用机器学习 模型指导下一步的试验进行了探讨和分析。

\section{1 线性回归预测}

线性回归算法使用最小二乘法来拟合得到式 2 中的权重系数和偏置，经过优化后的最终线性回归 方程为 


$$
\begin{gathered}
f(x)= \\
-0.79 x_{1}+0.61 x_{2}+0.14 x_{3}-0.18 x_{4}+0.91
\end{gathered}
$$

上式在训练集和测试集上的预测结果如图 3 所示, 其中, 圆点代表预测值, 三角形代表试验值, 两者 之间的差距由两数据点间线段绘制, 该结果可视化 方式在后续的两种算法中同样沿用。

○ 线性回归预测 $\Delta$ 试验值

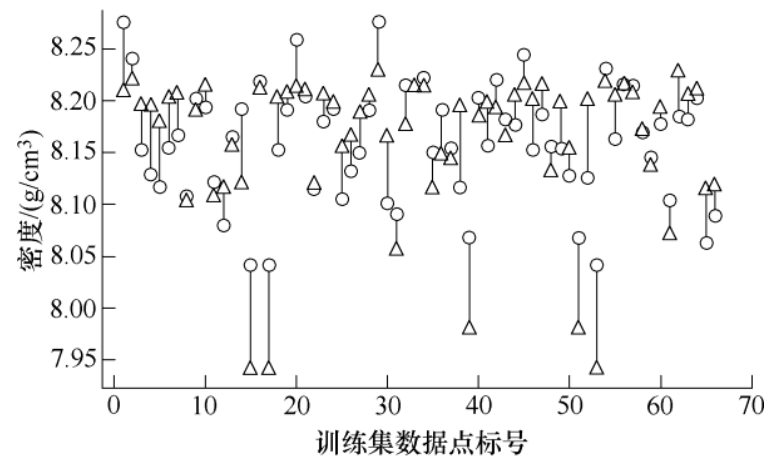

(a) 训练集

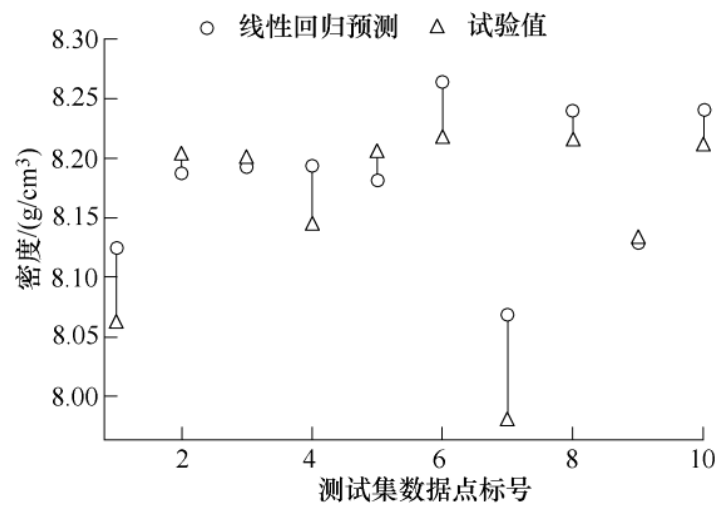

(b) 测试集

图 3 线性回归算法在数据集上的预测

由图 3 可得, 线性回归算法的预测准确率较低, 尤其是在低密度值情形下。究其原因, 一是因为该 模型的前提假设是基于特征量与响应之间是确定的 线性关系，该假设对于复杂非线性关系的拟合能力 不足。另一方面, 该模型没有可调的超参数, 因此, 模型本身的容量也很小, 不足以描述数据之间内在 的复杂关系。

\section{2 支持矢量回归预测}

支持矢量回归算法选择高斯核作为核函数形 式, 即

$$
\kappa\left(x_{i}, x_{j}\right)=\exp \left(-\frac{\left\|x_{i}-x_{j}\right\|^{2}}{2 \sigma^{2}}\right)
$$

式中, $\left\|x_{i}-x_{j}\right\|^{2}$ 可以看做两个特征矢量之间的平方 欧几里得距离, $\sigma$ 是核函数的带宽，控制函数的局 部作用范围, 通常使用另一个参数 $\gamma$ 来简化替代 $\sigma$, 表达式为 $\gamma=1 / 2 \sigma^{2}$ 。经过训练优化后, 得到模型中
的各个超参数数值分别为惩罚系数 $C=800$, 预测值 和实际值的允许偏差 $\varepsilon=0.08$ 和带宽系数 $\gamma=1.1$ 。

支持矢量回归的预测结果如图 4 所示，可以 看出, 该算法无论在训练集和测试集上都有很高 的准确率, 且无论在高密度值和低密度值时都吻 合很好, 说明算法已经学习到了低密度值时的特 征模式。这是因为支持矢量回归算法是一种非线 性映射关系，其利用内积核函数将特征内在的非 线性关系转换为高维空间的线性关系, 且惩罚系数 $C$ 、偏差 $\varepsilon$ 等超参数又保证了模型足够的容量以容 纳数据的复杂性。

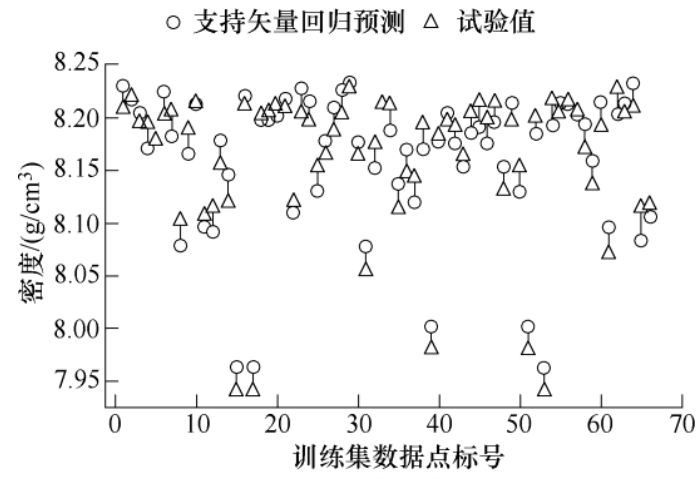

(a) 训练集

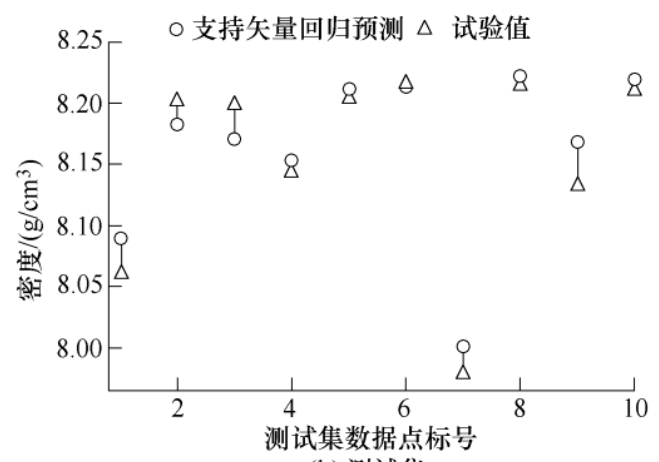

(b) 测试集

图 4 支持矢量回归算法在数据集上的预测

\section{3 神经网络预测}

神经网络模型是三种算法中超参数最多的算 法。首先, 对于网络结构, 需要提前定义隐藏层的 个数、单个隐藏层的神经元的个数、有无正则化、 激活函数和损失函数形式等参数; 再者, 对于训练 过程, 需要设定迭代次数、学习速率、权重和偏置 初始化方法、优化算法等参数。通过设定一系列不 同的超参数组合，对比获得其中最优的神经网络模 型如下。

（1）网络结构: 单个隐藏层, 该隐藏层的神经 元个数为 7 , 无正则化, 激活函数 Tanh, 损失函数 选择均方误差 $\sqrt{\frac{1}{n} \sum_{i=1}^{n}\left|y_{i}-y_{i}\right|}$ 。

（2）训练过程: 迭代次数 20000 , 初始学习速 
率 0.0001 , 使用 Xavier ${ }^{[17]}$ 初始化权重系数, 初始偏 置设为 0 , 优化算法为 $\operatorname{Adam}^{[18]}$ 。

该神经网络模型的预测结果如图 5 所示, 可以 看出, 模型精度较好, 对于高密度值和低密度值有 较好的预测, 但对于中间数值却反而有较大误差。 需要指出的一点是, 如前所述, 神经网络的超参数 众多, 这意味着其模型容量大, 即在训练集上训练 该算法时, 完全可以通过超参数调优将其在训练集 上的误差降低到极低程度 (甚至比支持矢量回归算 法误差更小); 但与此同时, 算法极易产生“过拟合”, 即在测试集上的性能很差。因为我们更看重的是模 型的泛化能力, 即预测 “未曾见过” 的新数据的能 力, 所以此处训练得到的模型兼顾了其在训练集和 测试集上的性能。

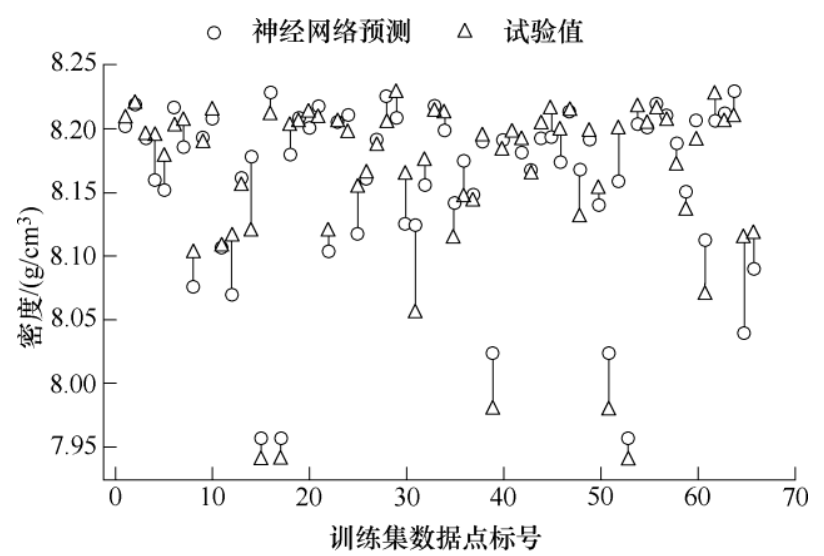

(a) 训练集

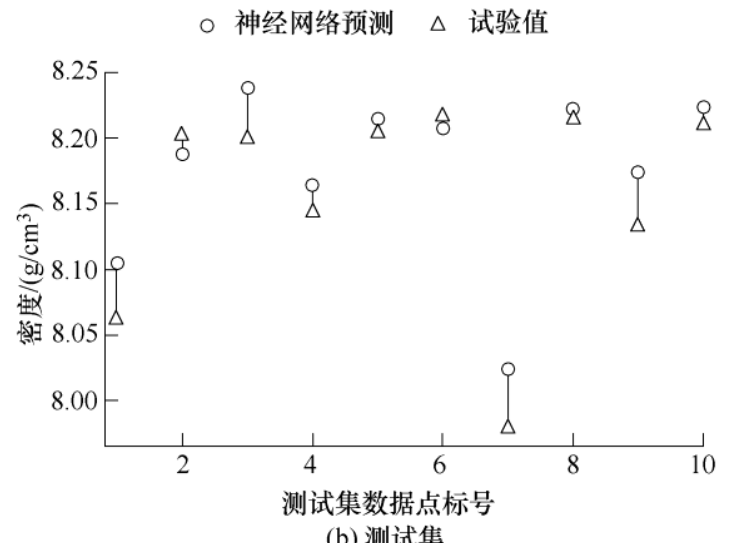

图 5 神经网络算法在数据集上的预测

\section{4 算法对比评价}

为了定量对比上述三种算法的预测能力, 这里 选择了三个性能指标来衡量模型的性能, 分别如下 所述。

（1）决定系数 $R^{2}$, 用来表征因变量的变异中有 多少百分比可由自变量来解释, 最佳分数为 1 , 表 明模型的参考价值很高, 最差为 0 , 表明模型完全 无参考价值

$$
\begin{aligned}
R^{2}(y, y) & =1-\frac{\sum_{i=1}^{n}\left(y_{i}-y_{i}\right)^{2}}{\sum_{i=1}^{n}\left(y_{i}-\bar{y}\right)^{2}} \\
\bar{y} & =\frac{1}{n} \sum_{i=1}^{n} y_{i}
\end{aligned}
$$

(2) 平均绝对误差 MAE，用来提供绝对误差损 失预期值，又称为 $\mathrm{L} 1$ 范数损失

$$
\operatorname{MAE}(y, y)=\frac{1}{n} \sum_{i=1}^{n}\left|y_{i}-y_{i}\right|
$$

(3) 均方误差 MSE，用来提供平方误差损失的 预期值，又称为 $\mathrm{L} 2$ 范数损失

$$
\operatorname{MSE}(y, y)=\frac{1}{n} \sum_{i=1}^{n}\left(y_{i}-y_{i}\right)^{2}
$$

三种机器学习算法作用于不同的数据集, 其相 对应的三种性能指标如表 2 所示。从决定系数指标 来看, 支持矢量回归算法在所有数据集上都能达到 0.9 以上, 说明该算法的解释性很好, 且非常可信; 与之对比的是, 神经网络算法的决定系数在 0.8 量 级，而线性回归算法甚至仅在 0.6 量级上，说明这 两种算法的可信赖程度较低。另外，从平均绝对误 差和均方误差这两种误差形式来看，支持矢量回归 的误差值都是最小, 说明该模型的拟合性能很好。 综上，所有的指标对比都表明，支持矢量回归是三

\begin{tabular}{|c|c|c|c|c|}
\hline 算法 & 数据集 & 决定系数 & 平均绝对误差 $(\%)$ & 均方误差 $(\%)$ \\
\hline \multirow{3}{*}{ 线性回归 } & 训练集 & 0.614 & 3.535 & 0.193 \\
\hline & 测试集 & 0.664 & 3.554 & 0.193 \\
\hline & 总集 & 0.622 & 3.538 & 0.193 \\
\hline \multirow{3}{*}{$\begin{array}{c}\text { 支持 } \\
\text { 矢量回归 }\end{array}$} & 训练集 & 0.928 & 1.736 & 0.036 \\
\hline & 测试集 & 0.925 & 1.787 & 0.043 \\
\hline & 总集 & 0.927 & 1.742 & 0.037 \\
\hline \multirow{3}{*}{ 神经网络 } & 训练集 & 0.878 & 1.846 & 0.061 \\
\hline & 测试集 & 0.857 & 2.446 & 0.082 \\
\hline & 总集 & 0.875 & 1.925 & 0.064 \\
\hline
\end{tabular}
种算法中性能最佳的算法，神经网络次之，而线性 回归算法最差。

表 2 三种机器学习算法在不同数据集上的性能对比

\section{5 应用机器学习模型指导试验}

如前所述，密度大小对于成形件的机械性能影 响显著：密度越大，材料的显微孔洞越少，使得制 件的机械性能越高。因此, 实际生产制造过程中, 总是期望尽可能得到密度最大的金属制件。应用上 述机器学习算法建立四种工艺参数与成形件密度之 间的数学模型后，即可应用该模型求解如下最优化 问题: 在给定的工艺参数约束条件下, 如何选择最 
优的工艺参数组合方案, 使得电子束熔化成形件的 密度最大。

通过合适的最优化算法求解该问题, 得到目标 参数空间后, 即可用于下一步的试验, 为探究最佳 质量的制件提供指导。具体地, 注意到机器学习算 法建立的数学模型没有显式的解析表达式, 因此无 法计算密度这一因变量对工艺参数自变量的一阶和 二阶导数, 因此这里可供调用的最优化算法主要是 一些智能算法, 如模拟退火、差分进化和遗传算法 等 ${ }^{[21]}$, 该部分的寻优求解工作以及后续的试验验证 将是本文的下一步延展。

\section{4 结论}

(1) 应用三种机器学习算法: 线性回归、支持 矢量回归和神经网络, 建立了电子束选区熔化增材 制造四种工艺参数(扫描速度、束流强度、基板温度 和粉层厚度)与成形件密度之间的数学关系。

(2) 支持矢量回归算法的准确度最高, 预测能 力最强, 同时模型的可解释性最好。

(3) 神经网络算法准确度较高, 但可调超参数 众多, 且物理意义不明确。

（4）线性回归算法的拟合效果最差, 其模型容 量不足，无法表示特征之间的非线性关系。

(5) 利用机器学习算法建立工艺参数与密度之 间的数学模型后, 还需进一步利用最优化算法寻找 得到最大密度的工艺参数组合。

\section{参 考 文 献}

[1] 汤慧萍, 王建, 逯圣路, 等. 电子束选区熔化成形技术 研究进展[J]. 中国材料进展, 2015(3): 49-59.

TANG Huiping, WANG Jian, LU Shenglu, et al. Research progress in selective electron beam melting[J]. Materials China, 2015(3): 49-59

[2] PRABHAKAR P, SAMES $\mathrm{W}$, DEHO $\mathrm{R}$, et al. Computational modeling of residual stress formation during the electron beam melting process for inconel 718[J]. Additive Manufacturing, 2015, 7: 83-91.

[3] MURR L. Metallurgy of additive manufacturing : Examples from electron beam melting[J]. Additive Manufacturing, 2015, 5: 40-53.

[4] MURR LE, GAYTAN SM, RAMIREZ DA, et al. Metal fabrication by additive manufacturing using laser and electron beam melting technologies[J]. Journal of Materials Science \& Technology, 2012，28(1): 1-14.

[5] 刘海涛, 赵万华, 唐一平. 电子束熔融直接金属成型工 艺的研究 [J]. 西安交通大学学报, 2007, 41(11):
1307-1310.

LIU Haitao, ZHAO Wanhua, TANG Yiping. Process investigation of direct metal fabrication based on electron beam melting[J]. Journal of Xi'an Jiaotong University, 2007, 41(11): 1307-1310.

[6] 颜永年, 齐海波, 林峰, 等. 三维金属零件的电子束选 区熔化成形[J]. 机械工程学报, 2007，43(6): 87-92.

YAN Yongnian, QI Haibo, LIN Feng, et al. Produced three-dimensional metal parts by electron beam selective melting[J]. Chinese Journal of Mechanical Engineering, 2007, 43(6): 87-92

[7] 周斌, 张婷, 林峰, 等. 电子束选区熔化成形 $\mathrm{ti} 6 \mathrm{a} 14 \mathrm{v}$ 和 3161 不锈钢叶轮体微观组织和力学性能的研究 [J]. 稀 有金属材料与工程，2018，47(1)：175-180.

ZHOU Bin, ZHANG Ting, LIN Feng, et al. Study of microstructures and mechanical properties of Ti6Al4V and 316L stainless steel impeller body by electron beam selective melting[J]. Rare Metal Materials and Engineering, 2018, 47(1): 175-180.

[8] SHEN X, YAO J, WANG Y, et al. Density prediction of selective laser sintering parts based on artificial neural network[M]. Berlin , Heidelberg : Springer Berlin Heidelberg, 2004.

[9] ZHANG W, MEHTA A, DESAI P S, et al. Machine learning enabled powder spreading process map for metal additive manufacturing $(\mathrm{AM})[\mathrm{C}] / /$ Solid Freeform Fabrication 2017: Proceedings of the 28th Annual International Solid Freeform Fabrication Symposium-An Additive Manufacturing Conference, August 7-9, 2017, Hilton Hotel Austin, Austin, Texas, USA: TMS, 2017: 1235-1249.

[10] 蔡从中, 裴军芳, 温玉锋, 等. 选择性激光烧结成型件 密度的支持矢量回归预测 [J]. 物理学报, 2009, 58(13): 8-S14.

CAI Congzhong, PEI Junfang, WEN Yufeng, et al. Density prediction of selective laser sintering parts based on support vector regression[J]. Acta Physics Sinica, 2009, 58(13): 8-S14.

[11] FLECK N A, SMITH R A. Effect of density on tensile strength, fracture toughness, and fatigue crack propagation behavior of sintered steel[J]. Powder Metallurgy, 1981, 24(3): 121-125.

[12] CHAWLA N, DENG X, MARRUCCI M, et al. Effect of density on the microstructure and mechanical behavior of powder metallurgy Fe-Mo-Ni steels[J]. Advances in Powder Metallurgy and Particulate Materials，2003，6: 7-257.

[13] ASTM. Standard test methods for density of compacted or sintered powder metallurgy (PM) products using archimedes' principle[S]. Philadelphia : ASTM 
International, 2009

[14] HE H, GARCIA E A. Learning from imbalanced data[J]. IEEE Transactions on Knowledge \& Data Engineering, 2009, 21(9): 1263-1284.

[15] ARLOT S, CELISSE A. A survey of cross-validation procedures for model selection[J]. Statistics Surveys, 2010, 4: 40-79.

[16] AKSOY S, HARALICK R M. Feature normalization and likelihood-based similarity measures for image retrieval[J]. Pattern Recognition Letters, 2001， 22(5): 563 - 582.

[17] PEDREGOSA F, GRAMFORT A, MICHEL V, et al. Scikit-learn: Machine learning in python[J]. J. Mach. Learn Res., 2012, 12(10): 2825-2830.

[18] PASZKE A, GROSS S, CHINTALA S, et al. Automatic dierentiation in pytorch[C]// 31st Conference on Neural Information Processing Systems, Long Beach, CA, USA: NIPS, 2017.

[19] HE K, ZHANG X, REN S, et al. Delving deep into rectifers: Surpassing human-level performance on imagenet classification[C]// Proceedings of the IEEE International Conference on Computer Vision. Washington, DC, USA : IEEE Computer Society, 2015: 1026-1034.

[20] KINGMA D P, BA J. Adam: A method for stochastic optimization[C]// International Conference on Learning Representations (ICLR), May 7-9, 2015, San Diego: Ithaca, NY: arXiv.org, 2015.

[21] CHONG E K P, ZAK S H. An introduction to optimization[M]. New York: John Wiley \& Sons, 2013.

作者简介: 元欣波(通信作者), 男, 1988 年出生, 博士后。主要研究方 向为智能制造。

E-mail: qixinbo@gmail.com

李长鹏, 男, 1977 年出生, 高级工程师。主要研究方向为智能制造。

E-mail: changpeng.li@siemens.com

林峰, 男, 1966 年出生, 教授。主要研究方向为增材制造和生物制造。

E-mail: linfeng@tsinghua.edu.cn

李勇, 男, 1962 年出生, 教授。主要研究方向为微细电加工工艺与装备、 微流控器件、超精密切削加工及其应用。

E-mail: liyong@mail.tsinghua.edu.cn 